\title{
GENERALIZED HELICES ON N-DIMENSIONAL RIEMANN-OTSUKI SPACES
}

\author{
Jeta ALO*
}

\begin{abstract}
In this paper the well-known properties of helices in Euclidian 3-space are extended to n-dimensional Riemann-Otsuki space. We define the infinitesimal deformations of curves in Riemann-Otsuki space and obtain the condition such that the given deformation of a curve defines a generalized Helix in this space.
\end{abstract}

Keywords: Riemann-Otsuki Spaces, infinitesimal deformations, generalized helices

*Makale Gönderim Tarihi: 07.05.2019 ; Makale Kabul Tarihi : 20.06.2019 Makale Türü: Araştırma DOI: $10.20854 /$ bujse. 561471

*Beykent University, Faculty of Sciences and Letters, Department of Mathematics (jeta@ beykent.edu.tr) 


\title{
N-BOYUTLU RIEMANN-OTSUKI UZAYLARINDA GENELLEŞTIRILMIŞ HELISLER
}

\author{
Jeta ALO*
}

ÖZ

$\mathrm{Bu}$ makalede, helis eğrilerinin 3 boyutlu Öklid uzayında bilinen özellikleri n-boyutlu Riemann Otsuki uzayında genişletilmiştir. Riemann-Otsuki uzayında eğrilerin sonsuz küçük deformasyonlarını tanımlayarak verilen bir deformasyonun bir genelleştirilmiş helisi tanımlaması için koşullar belirlenmiştir.

Anahtar Kelimeler: Riemann-Otsuki uzayları, sonsuz, küçük deformasyonlar, genelleştirilmiş helisler

*Makale Gönderim Tarihi: 07.05.2019 ; Makale Kabul Tarihi : 20.06.2019 Makale Türü: Araştırma DOI: 10.20854 /bujse.561471

*Beykent Üniversitesi, Fen Edebiyat Fakültesi, Matematik Bölümü (jeta@beykent.edu.tr) 


\section{INTRODUCTION}

As is known in 3-Dimensional Euclidean space, if there is a 1-1 correspondence between the points of two curves such that the tangent vectors to these corresponding points are parallel, then the first and second normals to theses points are parallel, too. Such curves are said to be deducible from each other by Combescure transformations.

Hayden [1] studied this property of curves in Riemannian space where he used the concept of "parallel" in the accepted sense for a Riemannian space. He studied the infinitesimal deformation which displaces the tangent parallely at each point. If it also displaces the 1-normal, 2-normal, $\ldots,(\mathrm{n}-1)$ normal parallely at each point then he called this transformation a "General Combescure Transformation (G. C. Transformation)". If every transformation of a curve which displaces its tangents is a G.C Transformation then it is said that the curve possesses the G.C. property and if every curve of a space possesses the G.C. property then it is said the space possess the G.C. property. He showed that the only Riemannian spaces which possess the G.C. property are flat spaces and he examined some special cases of deformations which displace the tangent parallely.

In this paper these properties of curves are studied in Riemann-Otsuki spaces.

\section{Riemann Otsuki Spaces}

Using the concept of tangent bundle of order 2 which is denoted by $\Im^{2}(M)$, Otsuki showed that the classical connections such as affine, projective and conformal connectiozns on manifolds can be considered from unificative standpoint [2-4] . Otsuki defined a general connection, as a cross section of the vector bundle $T(M) \otimes \mathrm{D}^{2}(M)$, where $D^{2}(M)$ is the dual vector bundle of $\Im^{2}(M)$ In local coordinates $u^{i}$, a general connection $\Gamma$ can be represented by.

$\Gamma=\partial u_{i} \otimes\left(P_{j}^{i} d^{2} u^{k}+\Gamma_{j k}^{i} d u^{i} \otimes d u^{k}\right)$,

and it can be written simply by $\Gamma=\left(P_{j}^{i}, \Gamma_{j k}^{i}\right)$ where $P_{j}^{i}$ are components of a tensor type $(1,1)$ which is denoted by $P=\lambda(\Gamma)$,and is called the principal endomorphism of $T(M)$. If $\mathrm{P}$ is an identity isomorphism of $T(M)$, i.e. $P_{j}^{i}=\delta_{j}^{i}$ then $\Gamma$ is an affine connection. Thereafter he defined the covariant derivative with respect to this connection

$$
\begin{aligned}
& V_{m_{1} \ldots m_{q} ; h}^{k_{1} \ldots k_{p}}=P_{i_{1}}^{k_{1}} \ldots P_{i_{p}}^{k_{p}} \frac{\partial V_{j_{1} \ldots j_{q}}^{i_{1}, i_{p}}}{\partial u^{h}} P_{m_{1}}^{j_{1}} \ldots P_{m_{q}}^{j_{q}} \\
& +\sum_{s=1}^{p} P_{i_{1}}^{k_{1}} \ldots P_{i_{s-1}}^{k_{s-1}} \Gamma_{i_{s} h}^{k_{s}} P_{i_{s+1}}^{k_{s+1}} \ldots P_{i_{p}}^{k_{p}} \bigvee_{j_{1} \ldots j_{g}}^{i_{1} \ldots i_{p}} P_{m_{1}}^{j_{1}} \ldots P_{m_{q}}^{j_{q}}
\end{aligned}
$$

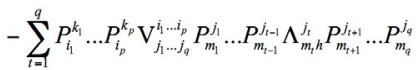

where $\Lambda_{i h}^{j}=\Gamma_{i n}^{j} \frac{\partial P_{i}^{\prime}}{\partial u^{h}}$.Moreover, Otsuki showed that the product of a tensor of type $(1,1)$ and the general connection is a general connection, too. These connections denoted by ' $\Gamma=Q \Gamma=\left(Q_{k}^{i} P_{j}^{k}, Q_{k}^{i} \Gamma_{j h}^{k}\right)$ and $" \Gamma=\Gamma Q=\left(P_{k}^{i} Q_{j}^{k}, \Lambda_{k s}^{i} Q_{j}^{k}\right)$ are called, respectively, the contravariant and the covariant part of the connection $\Gamma$,

$$
\begin{aligned}
\Gamma & =\partial u_{i} \otimes\left(Q_{k}^{i} P_{j}^{k} d^{2} u^{j}+Q_{k}^{i} \Gamma_{j h}^{k} d u^{j} \otimes d u^{h}\right) \\
& =\partial u_{i} Q_{k}^{i} \otimes\left(P_{j}^{k} d^{2} u^{j}+\Gamma_{j h}^{k} d u^{j} \otimes d u^{h}\right)=Q \Gamma \\
& \Gamma=\partial u_{i} \otimes\left(d\left(P_{k}^{i} Q_{j}^{k} d u^{j}\right)+\Lambda_{k h}^{i} Q_{j}^{k} d u^{h} \otimes d u^{j}\right)=\Gamma Q .
\end{aligned}
$$

If $\Gamma$ is a regular general connection and $Q=P^{-1}$ we get

$$
\begin{aligned}
& \Gamma=P^{-1} \Gamma=\left(\delta_{j}^{i}, Q_{k}^{i} \Gamma_{j h}^{k}\right)=\left(\delta_{j}^{i}, \Gamma_{j h}^{i}\right) \\
& \Gamma=\Gamma P^{-1}=\left(\delta_{j}^{i}, \Lambda_{k h}^{i} Q_{j}^{k}\right)=\left(\delta_{j}^{i}, " \Gamma_{j h}^{i}\right)
\end{aligned}
$$

i.e. $\Gamma$ and $\Gamma$ are affine connections which are called respectively contravariant and covariant part of a regular general connection Г.Then T.Otsuki defined a basic covariant differential by

$\bar{D} V_{j_{1} \ldots j_{q}}^{i_{1} \ldots i_{p}}=V_{j_{1} \ldots j_{q} / h^{\prime}}^{i_{1}, i_{p}} d u^{h}$

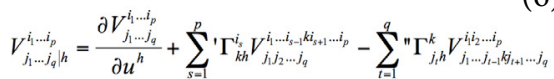

and showed that

$$
V_{j_{1} \ldots j_{q} ; m}^{i_{i} \ldots i_{p}}=P_{k_{1}}^{i_{1}} \ldots P_{k_{p}}^{i_{p}} V_{h_{1} \ldots h_{q} \mid m}^{k_{1} \ldots k_{p}} P_{j_{1}}^{h_{1}} \ldots P_{j_{q}}^{h_{q}} .
$$

For a general connection $\Gamma=\left(P_{j}^{i}, \Gamma_{j h}^{i}\right)$ and an identity isomorphism I we have

$\frac{D \delta_{j}^{i}}{d s}=\left(\Gamma_{h k}^{i} P_{j}^{h}-P_{h}^{i} \Lambda_{j k}^{h}\right) \frac{d x^{k}}{d s}$

and when $\Gamma$ is regular we get

$\frac{D \delta_{j}^{i}}{d s}=\left(' \Gamma_{j k}^{i}-" \Gamma_{j k}^{i}\right) \frac{d x^{k}}{d s}$

which does not necessarily vanish. From the equations (5) we find

$\frac{\partial P_{j}^{i}}{\partial u^{h}}+" \Gamma_{k h}^{i} P_{j}^{k}-P_{k}^{i} \Gamma_{j h}^{k}=0$

the Otsuki equation which gives the relationship between the covariant and contravariant parts of a general connection $\Gamma$. Riemann-Otsuki spaces are characterized by a Riemannian metric associated with general connection concept defined by T.Otsuki. 
A general connection determined by the equation $\nabla_{k} g_{i j}=0$ defines a Riemann-Otsuki space which will be denoted by $\left(R-O_{n}\right)$ [5-6]. This space, as defined by A.Moor [7-8] is a special case of a Weyl-Otsuki space.

The Frenet Formulas for the Riemann-Otsuki space with respect to' $D$ zapplied to contravariant components of the tangent and normal vectors $\quad(' D \equiv \bar{D}$ for contravariant vectors), Nadj [9], are:

$\bar{D} V_{(\alpha)}^{i}={ }^{\prime} D V_{(\alpha)}^{i}=\kappa_{\alpha} V_{(\alpha+1)}^{i}-\kappa_{\alpha-1} V_{(\alpha-1)}^{i}+V_{(\alpha)}^{q} \bar{D} \delta_{q}^{i}$

$\left(\alpha=1,2, \ldots, n ; \kappa_{0}=0, \kappa_{n}=0\right)$

\section{Parallel tangent deformations}

Let $\left(R-O_{n}\right)$, be an n-dimensional Riemann-Otsuki space and $\Gamma=\left(P_{j}^{i}, \Gamma_{j k}^{i}\right)$ a general connection with $\nabla_{k} g_{i j}=0$ Let

$x^{\lambda}=x^{\lambda}(s)$

be a curve on $\left(R-O_{n}\right)$ with an arc length $\mathrm{s}$, and let $\delta x^{\lambda} \equiv \varepsilon \xi^{\lambda}$ be an infinitesimal deformation at each point of this curve, where $\varepsilon$ is an infinitesimal constant and $\xi^{\lambda}$ is vector field along $C$ with $\xi \equiv \sqrt{g_{\mu \nu} \xi^{\mu} \xi^{\nu}}$ Let $\bar{x}^{\lambda}=x^{\lambda}+\varepsilon \xi^{\lambda}$

be an infinitesimal deformation of the curve (10). The infinitesimal deformations in Riemannian n- space are studied by Hayden [1] and Yano [10-11].

Let $V_{(1)}^{\lambda}, \ldots, V_{(n)}^{\lambda}$ be tangent, 1-normal, 2-normal,..., (n-1)-normal vectors, respectively at any point $\mathrm{P}$ of the curve $\mathrm{C}$ and $\bar{V}_{(1)}^{\lambda}, \ldots, \bar{V}_{(n)}^{\lambda}$ the tangent, 1-normal,...,(n-1)normal, respectively at the corresponding point $\bar{P}$ of $\bar{C}$. Then

$\bar{V}_{(1)}^{\lambda}=\left(V_{(1)}^{\lambda}+\varepsilon \frac{d \xi^{\lambda}(s)}{d s}\right) \frac{d s}{d s}$

Let $* V^{\lambda}(t)$ be the vector at $\bar{P}$ parallel to the vector $V_{(1)}^{\lambda}$ at the point $P$. Since for parallel vector fields

$\frac{d v^{\lambda}}{d t}+{ }^{\prime} \Gamma_{\mu v}^{\lambda} v^{\mu} \frac{d x^{v}}{d t}=0$

holds, we get

$V_{(1)}^{\lambda}(\bar{x})={ }^{*} V_{(1)}^{\lambda}\left(x+\varepsilon \xi^{\lambda}\right)=V_{(1)}^{\lambda}(x)+\varepsilon \frac{d V_{(1)}^{\lambda}(x)}{d x^{\omega}} \xi^{\omega}=V_{(1)}^{\lambda}(x)-\varepsilon^{\prime} \Gamma_{\mu \nu}^{\lambda} V_{(1)}^{\mu} \xi$

In this case $\bar{V}_{(1)}^{\lambda}-* V_{(1)}^{\lambda}$ is an infinitesimal vector which is denoted by $\delta V_{(1)}^{\lambda}$ and

$\Delta V_{(1)}^{\lambda}=\lim _{\delta s \rightarrow 0} \frac{\delta V_{(1)}^{\lambda}}{\delta s}=\lim _{\delta s \rightarrow 0} \frac{1}{\delta s}\left(\bar{V}_{(1)}^{\lambda}(\bar{x})-{ }^{*} V_{(1)}^{\lambda}(\bar{x})\right)$

where $\delta s \equiv \sqrt{g_{\mu \nu} \delta x^{\mu} \delta x^{\nu}}=\sqrt{g_{\mu v}\left(\bar{x}^{\mu}-x^{\mu}\right)\left(\bar{x}^{v}-x^{v}\right)}=\sqrt{g_{\mu v} \varepsilon \xi^{\mu} \varepsilon \xi^{\nu}}=\varepsilon \xi$.

From (12) and (13) we find,

$\delta V_{(1)}^{\lambda}=\bar{V}_{(1)}^{\lambda}-{ }^{\prime \prime} V_{(1)}^{\lambda}=V_{(1)}^{\lambda}\left(\frac{d s}{d \bar{s}}-1\right)+\varepsilon\left(\frac{d \xi^{\lambda}}{d s} \frac{d s}{d \bar{s}}+{ }^{\prime} \Gamma_{\mu v}^{\lambda} V_{(1)}^{\mu} \xi^{v}\right)$ to the first order. We find $\frac{d s}{d s}$ from $\bar{g}_{\mu \nu} \bar{V}_{(1)}^{\mu} \bar{V}_{(1)}^{v}=1$
where

$$
\begin{aligned}
& \bar{g}_{\mu \nu}=g_{\mu \nu}+\frac{\partial g_{\mu v}}{\partial x^{\omega}}\left(\bar{x}^{\omega \nu}-x^{\omega \nu}\right)=g_{\mu v}+\varepsilon \xi^{\omega \nu}\left(g_{\mu \alpha} " \Gamma_{v \omega}^{\alpha}+g_{v \alpha} " \Gamma_{\mu \omega}^{\alpha}\right) \\
& d \bar{s}^{2}=\bar{g}_{\mu v} d \bar{x}^{\mu} d \bar{x}^{v} \\
& d \bar{s}^{2}=\left(g_{\mu v}+\varepsilon g_{\mu v, \omega} \xi^{\omega}\right)\left(d x^{\mu}+\varepsilon d \xi^{\mu}\right)\left(d x^{\nu}+\varepsilon d \xi^{v}\right) \\
& d \bar{s}^{2}=d s^{2}+\left(g_{\mu v, \omega} \xi^{\omega}+2 g_{a v} \xi_{, \mu}^{\alpha}\right) d x^{\mu} d x^{v} \varepsilon \\
& d \bar{s}^{2}=d s^{2}+\left\{\left(g_{\mu \alpha} " \Gamma_{v \omega}^{\alpha}+g_{v \alpha} " \Gamma_{\mu \omega}^{\alpha}\right) \xi^{\omega}+2 g_{\alpha v}\left(\xi_{; \mu}^{\alpha}-\Gamma^{\prime \mu \mu} \xi^{\omega}\right)\right\} d x^{\mu} d x^{v} \\
& d \bar{s}^{2}=d s^{2}+2 \varepsilon g_{\alpha \nu}\left\{\xi_{; \mu}^{\alpha}-\delta_{\mu \mid \omega}^{\alpha} \xi^{\omega}\right\} d x^{\mu} d x^{\nu} \\
& \left(\frac{d \bar{s}}{d s}\right)^{2}=1+2 \varepsilon g_{\alpha v}\left\{\xi_{; \mu}^{\alpha}-\delta_{\mu \mid \omega}^{\alpha} \xi^{\omega}\right\} \frac{d x^{\mu}}{d s} \frac{d x^{v}}{d s} \\
& =1+2 \varepsilon\left(\frac{\bar{D} \xi^{\alpha}}{d s}-\frac{\bar{D} \delta_{\omega}^{\alpha}}{d s} \xi^{\omega}\right) V_{(1) \alpha}
\end{aligned}
$$

If we put

$$
\left(\frac{\bar{D} \xi^{\lambda}}{d s}-\frac{\bar{D} \delta_{\omega}^{\lambda}}{d s} \xi^{\omega}\right) V_{(1) \lambda}=\phi
$$

so that $\phi+\frac{\bar{D} \delta_{\omega}^{\lambda}}{d s} \xi^{\omega} V_{(1) \lambda}$ is the component of $\frac{\bar{D} \xi^{\lambda}}{d s}$ along the tangent, we find

$$
\begin{aligned}
\left(\frac{d \bar{s}}{d s}\right)^{2} & =1+2 \varepsilon g_{\alpha \nu}\left\{\xi_{; \mu}^{\alpha}-\delta_{\mu(\omega}^{\alpha} \xi^{\omega}\right\} \frac{d x^{\mu}}{d s} \frac{d x^{v}}{d s} \\
& =1+2 \varepsilon\left(\frac{\bar{D} \xi^{\alpha}}{d s}-\frac{\bar{D} \delta_{\omega}^{\alpha}}{d s} \xi^{\omega}\right) V_{(1) \alpha}=1+2 \varepsilon \phi \\
\frac{d \bar{s}}{d s} & =1+\varepsilon \phi \\
\frac{d s}{d \bar{s}} & =1-\varepsilon \phi
\end{aligned}
$$

and writing this equation in (15) we get

$$
\delta V_{(1)}^{\lambda}=-\varepsilon \phi V_{(1)}^{\lambda}+\varepsilon\left(\frac{d \xi^{\lambda}}{d s}+\Gamma_{\mu \nu}^{\lambda} V_{(1)}^{\mu} \xi^{v}\right)=\varepsilon\left(\frac{\bar{D} \xi^{\lambda}}{d s}-\phi V_{(1)}^{\lambda}\right)
$$

and

$$
\Delta V_{(1)}^{\lambda}=\lim _{\delta s \rightarrow 0} \frac{\delta V_{(1)}^{\lambda}}{\delta s}=\lim _{\delta s \rightarrow 0} \frac{\varepsilon}{\delta s}\left(\frac{\bar{D} \xi^{\lambda}}{d s}-\phi V_{(1)}^{\lambda}\right)=\frac{1}{\xi}\left(\frac{\bar{D} \xi^{\lambda}}{d s}-\phi V_{(1)}^{\lambda}\right)
$$

The condition for $\xi^{\lambda}$ to be the deformation which displaces the tangents parallelly so called "parallel tangent deformation" is $\Delta V_{(1)}^{\lambda}=0$ at every point of C. From (19) we find the conditions

$$
\frac{\bar{D} \xi^{\lambda}}{d s}=\phi V_{(1)}^{\lambda}
$$

and

$$
\frac{\bar{D} \xi^{\lambda}}{d s} V_{(\alpha) \lambda}=0, \quad(\alpha=2,3, \ldots, n)
$$


Writing this condition in (16) we find

$$
\frac{\bar{D} \delta_{\omega}^{\lambda}}{d s} \xi^{\omega} V_{(1) \lambda}=0
$$

the condition that $\xi{ }^{\lambda}$ is a parallel tangent deformation in Riemann-Otsuki spaces.

\section{Generalized Helices in Riemann-Otsuki} Spaces

Let $\xi^{\lambda}$ be a deformation such that

$\Delta V_{(1)}^{\lambda}=\frac{1}{\xi} \frac{\bar{D} \delta_{\beta}^{\lambda}}{d s} \xi^{\beta}$

This vector can be written in the terms of tangents and normals as

$$
\xi^{\lambda}=\sum_{\alpha=1}^{n} c_{\alpha} V_{(\alpha)}^{\lambda}
$$

where c's are scalar functions of s. By using the Frenet Formula's we find

$$
\begin{aligned}
\frac{\bar{D} \xi^{\lambda}}{d s} & =\sum_{\alpha=1}^{n}\left(c_{\alpha} \frac{\bar{D} V_{(\alpha)}^{\lambda}}{d s}+\frac{d c_{\alpha}}{d s} V_{(\alpha)}^{\lambda}\right) \\
& =\sum_{\alpha=1}^{n}\left(c_{\alpha}\left(\kappa_{\alpha} V_{(\alpha+1)}^{\lambda}-\kappa_{\alpha-1} V_{(\alpha-1)}^{\lambda}+V_{(\alpha)}^{\beta} \frac{\bar{D} \delta_{\beta}^{\lambda}}{d s}\right)+\frac{d c_{\alpha}}{d s} V_{(\alpha)}^{\lambda}\right) \quad(24) \\
& =\sum_{\alpha=1}^{n}\left(c_{\alpha-1} \kappa_{\alpha-1}+\frac{d c_{\alpha}}{d s}-c_{\alpha+1} \kappa_{\alpha}\right) V_{(\alpha)}^{\lambda}+\frac{\bar{D} \delta_{\beta}^{\lambda}}{d s} \xi^{\beta} \quad\left(\kappa_{0}=\kappa_{n}=0\right)
\end{aligned}
$$

Using (24) in equations (16) and (19) we get

$$
\begin{aligned}
& \phi \equiv\left(\frac{\bar{D} \xi^{\lambda}}{d s}-\frac{\bar{D} \delta_{\beta}^{\lambda}}{d s} \xi^{\beta}\right) V_{(1) \lambda} \\
& =\left(\sum_{\alpha=1}^{n}\left(c_{\alpha-1} \kappa_{\alpha-1}+\frac{d c_{\alpha}}{d s}-c_{\alpha+1} \kappa_{\alpha}\right) V_{(\alpha)}^{\lambda}+\frac{\bar{D} \delta_{\beta}^{\lambda}}{d s} \xi^{\beta}-\frac{\bar{D} \delta_{\beta}^{\lambda}}{d s} \xi^{\beta}\right) V_{(1) \lambda} \\
& =\left(\frac{d c_{1}}{d s}-c_{2} \kappa_{1}\right)
\end{aligned}
$$

and

$$
\begin{aligned}
\Delta V_{(1)}^{\lambda} & =\frac{1}{\xi}\left(\frac{\bar{D} \xi^{\lambda}}{d s}-\phi V_{(1)}^{\lambda}\right) \\
& =\frac{1}{\xi}\left(\begin{array}{l}
\left.\sum_{\alpha=1}^{n}\left(c_{\alpha-1} \kappa_{\alpha-1}+\frac{d c_{\alpha}}{d s}-c_{\alpha+1} \kappa_{\alpha}\right) V_{(\alpha)}^{\lambda}+\frac{\bar{D} \delta_{\beta}^{\lambda}}{d s} \xi^{\beta}\right) \\
-\left(\frac{d c_{1}}{d s}-c_{2} \kappa_{1}\right) V_{(1)}^{\lambda}
\end{array}\right) \\
& =\frac{1}{\xi}\left(\sum_{\alpha=2}^{n}\left(c_{\alpha-1} \kappa_{\alpha-1}+\frac{d c_{\alpha}}{d s}-c_{\alpha+1} \kappa_{\alpha}\right) V_{(\alpha)}^{\lambda}+\frac{\bar{D} \delta_{\beta}^{\lambda}}{d s} \xi^{\beta}\right)
\end{aligned}
$$

respectively.

Using the condition given in (23) we get

$$
\sum_{\alpha=2}^{n}\left(c_{\alpha-1} \kappa_{\alpha-1}+\frac{d c_{\alpha}}{d s}-c_{\alpha+1} \kappa_{\alpha}\right) V_{(\alpha)}^{\lambda}=0
$$

and from here,

$\frac{d c_{\alpha}}{d s}=c_{\alpha+1} \kappa_{\alpha}-c_{\alpha-1} \kappa_{\alpha-1}, \alpha=2,3, \ldots, n$

These are $\mathrm{n}-1$ equations of $\mathrm{n} c_{1}, \ldots, c_{n}$ scalar functions such that one of $c_{i}$ 's can be arbitrary defined. From (26) we find

$$
\begin{aligned}
& \frac{d c_{2}}{d s}=c_{3} \kappa_{2}-c_{1} \kappa_{1}, \\
& \frac{d c_{3}}{d s}=c_{4} \kappa_{3}-c_{2} \kappa_{2},
\end{aligned}
$$

$\frac{d c_{n-1}}{d s}=c_{n} \kappa_{n-1}-c_{n-2} \kappa_{n-2}$,

$\frac{d c_{n}}{d s}=-c_{n-1} \kappa_{n-1}$

If $c_{n}=0$ then for $\kappa_{n-1} \neq 0$ we find $c_{n-1}=0$ and from here $\quad c_{n-2}=\ldots=c_{2}=c_{1}=0 \quad$ which means that the detormation $\xi^{\lambda}=0$.

Now, let's suppose that $c_{\alpha}$ 's are constant. Then from (27) we get

$$
\begin{aligned}
& c_{3} \kappa_{2}-c_{1} \kappa_{1}=0, \\
& c_{4} \kappa_{3}-c_{2} \kappa_{2}=0, \\
& \cdots \cdots \cdots \cdots \cdots \cdots \cdots \cdots \cdots \cdots \cdots \cdots \cdots \cdots \cdots \cdots \cdots \cdots \cdots \\
& c_{n-1} \kappa_{n-2}-c_{n-3} \kappa_{n-3}=0, \\
& c_{n} \kappa_{n-1}-c_{n-2} \kappa_{n-2}=0, \\
& -c_{n-1} \kappa_{n-1}=0,
\end{aligned}
$$

and hence $c_{n-1}=0$, and

$c_{n-3}=c_{n-5}=\ldots=c_{2}=0$ if $\mathrm{n}$ is odd,

$c_{n-3}=c_{n-5}=\ldots=c_{1}=0$ if $\mathrm{n}$ is even and from here

$\frac{\kappa_{n-1}}{\kappa_{n-2}}=\frac{c_{n-2}}{c_{n}}=$ const. $, \ldots, \frac{\kappa_{2}}{\kappa_{1}}=$ const if $\mathrm{n}$ is odd,

$\frac{\kappa_{n-3}}{\kappa_{n-4}}=\frac{c_{n-4}}{c_{n-2}}=$ const. $, \ldots, \frac{\kappa_{3}}{\kappa_{2}}=$ const $\quad$ if $\mathrm{n}$ is even

For a Euclidian 3-space this curve is a helix, Hayden in [1] called this curvea "generalized helix" in Riemanniann-space.

In Riemann-Otsuki space, a "generalized helix" is defined by a deformation which satisfies the condition

$\Delta V_{(1)}^{\lambda}=\frac{1}{\xi} \frac{\bar{D} \delta_{\beta}^{\lambda}}{d s} \xi^{\beta}$

where $\xi^{\lambda}=\sum_{\alpha=1}^{n} c_{\alpha} V_{(\alpha)}^{\lambda} \quad$ with constant c's. This deformation must be in the $\left(\frac{1}{2} n+\frac{1}{2}\right)$ - space defined by $V_{(n)}^{\lambda}, V_{(n-2)}^{\lambda}, \ldots, V_{(1)}^{\lambda}$ if $\mathrm{n}$ is odd or in the $\frac{1}{2} n$ - space defined by $V_{(n)}^{\lambda}, V_{(n-2)}^{\lambda}, \cdots, V_{(2)}^{\lambda}$ if $\mathrm{n}$ is even. 


\section{BEYKENT ÜNIVERSITESI FEN VE MÜHENDISLIK BILIMLERI DERGISI \\ CILT SAYI:12/1 \\ www.dergipark.gov.tr}

\section{REFERENCES}

[1] HAYDEN, H.A., 1931, Deformation of a curve in a Riemannian n-space which displace certain vectors parallelly at each point, Proc. London Math. Soc. (2), 32, 321-336.

[2] OTSUKI, T., 1958, Tangent Bundles of Order 2 and General Connections, Math. J.

Okayama Univ., 8, 143-179.

[3] OTSUKI, T., 1960, On General Connections I, Math. J. Okayama Univ., 9, 99-164.

[4] OTSUKI, T., 1961, On General Connections II, Math. J. Okayama Univ., 10, 113-124

[5] Nadj, Dj.F.,1981, On Subspaces of Riemann-Otsuki Space, Publ. de 1'Inst. Math Beograd, 30 (44), 53-58

[6] Nadj, Dj.F.,1981, On the Orthogonal Spaces of the Subspaces of a Riemann-Otsuki Space , Zbornik Radova PMF Novi Sad, 11, 201-208

[7] MOOR, A., 1978, Otsukische Übertragung mit Rekurrentem Masstensor, Acta Sci.Math., 40, 129142.

[8] MOOR, A., 1979, Über die Veranderung der Lange der Vektoren in Weyl-Otsukischen Raumen, Acta Sci. Math., 41, 173-185

[9] NADJ, Dj.F., 1986 The Frenet Formula of Riemann-Otsuki space, Zbornik Radova PMF Novi Sad, 16, (1), 95-106.

[10] YANO, K., ADATI, T., 1944, Paralel tangent deformations, concircular transformation and concurrent vector field, Proc. Imp. Acad., 20, No3, 123-127

[11] YANO, K.,Kazuo, T., Yasuro, T.,1948, On infinitesimal deformations of curves in spaces with linear connection, Jap, J, Math., Volume 19, 433-477. 